\title{
Can Serum Cortisol Be Used To Monitor Patients With COVID-19?
}

\author{
Ashutosh Kumar, ${ }^{1,2}$ Chiman Kumari, ${ }^{1,3}$ Vikas Pareek, ${ }^{1,4}$ and Ravi K Narayan ${ }^{1,2}$ \\ 1. Etiologically Elusive Disorders Research Network, New Delhi, India; 2. Department of Anatomy, All India Institute of Medical Sciences, Patna, India; \\ 3. Department of Anatomy, Postgraduate Institute of Medical Education and Research, Chandigarh, India; 4. National Brain Research Center, Manesar, Haryana, India
}

DOI: https://doi.org/10.17925/USE.2020.16.2.63

$\mathrm{T}$ herapeutic administration of steroids (or corticosteroids) in patients with coronavirus disease (COVID-19) is controversial owing to risk of adrenal insufficiency or immunosuppression from prolonged use. A recent clinical trial report presenting preliminary data suggested survival benefits of low-dose dexamethasone in patients with severe COVID-19. Additionally, there is evidence that severe acute respiratory syndrome coronavirus-2 (SARS-CoV-2)-mediated pathogenetic mechanisms can involve the adrenal gland, and can therefore influence endogenous production of steroids, mainly cortisol. Cortisol is an output hormone of the hypothalamic-pituitary-adrenal axis-the chief endocrine axis maintaining physiological homeostasis. Despite the known dangers of possible endocrine disruption, there has been very little discussion in the literature regarding monitoring of serum cortisol concentrations in patients with COVID-19. In this article, we briefly discuss existing and emerging empirical evidence in favor of monitoring serum cortisol concentrations in patients with severe CoVID-19 in terms of improving survival outcomes as well as facilitating better therapeutic management.

\section{Keywords}

ACE2, adrenal gland, COVID-19, SARS-COV-2, steroids

Disclosures: Ashutosh Kumar, Chiman Kumari, Vikas Pareek, and Ravi K Narayan have no financial or non-financial relationships or activities to declare in relation to this article.

Review Process: Double-blind peer review. Compliance with Ethics: This article is an opinion piece and does not report on new clinical data, or any studies with human or animal subjects performed by any of the authors.

Authorship: The named authors meet the International Committee of Medical Journal Editors (ICMJE) criteria for authorship of this manuscript, take responsibility for the integrity of the work as a whole, and have given final approval for the version to be published.

Access: This article is freely accessible at touchENDOCRINOLOGY.com (c) Touch Medical Media 2020

Received: June 21, 2020

Accepted: August 24, 2020

Published Online: October 5, 2020

Citation: US Endocrinology. 2020;16(2):63-4

Corresponding Author: Ashutosh Kumar,

Department of Anatomy, All India Institute of Medical

Sciences, Phulwari Sharif, Patna, Bihar 801507, India.

E: drashutoshkumar@aiimspatna.org

Support: No funding was received in

the publication of this article.
Management of patients with severe COVID-19 remains a challenge for physicians. In severe cases of COVID-19, a hyperactive innate immune response characterized by high serum levels of proinflammatory markers, known as a 'cytokine storm', is characteristically present. The cytokine storm induces fulminant pulmonary inflammation known as acute respiratory distress syndrome, and subsequently systemic inflammation, which can lead to multi-organ failure.

The use of systemic steroids (or corticosteroids) in managing inflammation in COVID-19 has been controversial. There is a theoretical risk of worsening of lymphocytopenia - a key feature in patients with severe COVID-19-with systemic use of steroids. ${ }^{2}$ Apart from that, existing co-morbidities and other side effects associated with steroid administration, such as myopathy or muscle wasting, psychiatric disturbances, impaired wound healing, hyperglycemia and hypernatremia, and adrenal insufficiency, may be limiting factors for their prolonged use in critically ill patients with COVID-19. ${ }^{3}$

The principal steroid produced in the body is cortisol, which is the output hormone of the hypothalamic-pituitary-adrenal (HPA) axis-the central regulator of physiological homeostasis. ${ }^{4} \mathrm{HPA}$ axis dysregulation and hypercortisolemia in patients with severe COVID-19 can also be caused by acute physiological stress induced by severe illness and ventilators. ${ }^{5}$ Free serum cortisol plays a key role in potential dysfunction of the HPA axis response in severely ill patients. Reduced serum protein binding and persistent suppression of metabolic degradation, resulting in increased free serum cortisol concentrations, are the suggested mechanisms behind acute stress-caused hypercortisolemia in these patients.

Hypercortisolemia can cause dysregulation of the physiological innate immune response against viral infections, which is a known phenomenon in the development of viral respiratory diseases including COVID-19.?

A recent study by Tan et al. found increased serum cortisol concentrations were associated with higher mortality in patients with COVID-19 in general. The investigators noted that patients with COVID-19 mounted an acute cortisol stress response that was significantly higher than that in individuals without COVID-19. A doubling of cortisol concentration was associated with a significant $42 \%$ increase in the hazard of mortality after adjustment for age, the presence of comorbidities, and laboratory tests. ${ }^{8}$ 
Surprisingly, to our knowledge, to date a virus-mediated mechanism altering physiological cortisol regulation has not been taken into consideration in patients with COVID-19. Severe acute respiratory syndrome coronavirus-2 (SARS-CoV-2), the causative pathogen for this disease has been shown to bind to angiotensin-converting enzyme 2 (ACE2) protein in human epithelial cells, which facilitates its entry into the cells. ${ }^{9}$ ACE2 was also a cell entry receptor for SARS-CoV-1, the causative agent of the SARS pandemic of 2002. ${ }^{9}$ For ACE2-mediated host cell entry of the SARS viruses, co-expression of proteases, such as transmembrane protease serine 2 (TMPRSS2) or cathepsin-L (CTSL), are essential. ${ }^{9}$ ACE2 and TMPRSS2/CTSL are known to be expressed in secretory cells of the adrenal gland. ${ }^{10,11}$ These viral cell entry receptors are also expressed in vascular endothelium and are known to be secreted into the blood circulation. ${ }^{10,11}$ As we know that the adrenal gland is highly vascular, it can get infected by SARS-CoV-2 reaching the gland through blood vessels. Virus-mediated adrenal cortex injury may cause hypocortisolemia, which will necessitate exogenous steroid support in patients with COVID-19.12-14 The laboratory data, currently available in patients with COVID-19, do not suggest any obvious adrenal insufficiency in the acute settings; ${ }^{8}$ however, the same cannot be denied in the long-term, as was noted in patients with SARS. ${ }^{13}$

The results of randomized controlled trials of exogenous steroid use in critically ill patients varies widely across studies. Although a meta-analysis of these trials confirmed the marginal benefits of a low-dose, long course $(\sim 7$ days) steroid regimen in reducing short-term (28 days) mortality, no significant improvement could be found in long-term (90 days) mortality. ${ }^{15}$ Of note, the recently declared preliminary results of the large RECOVERY trial (Randomised Evaluation of COVID-19 Therapy; ClinicalTrials.gov identifier: NCT04381936) in the UK indicated a survival benefit of steroids if used in low doses. Investigators used $6 \mathrm{mg}$ of dexamethasone once daily for 10 days. The results showed a one-third and one-fifth reduction in 28-day mortality in patients on ventilator and oxygen support, respectively; however, it had no effect in those who were not receiving any respiratory support. ${ }^{16}$ Currently, there are no study reports available that can confirm the benefits of low-dose steroids on long-term (90 days) mortality in patients with COVID-19.

Reasons for the noted survival benefits of the low-dose dexamethasone in critically ill patients with COVID-19 are currently unexplained. A meta-analysis that examined the effects of steroids during sepsis in patients under intensive care, concluded that dose and severity of the illness are the deciding factors in the survival benefit relating to steroids. ${ }^{17}$ The paradoxical benefits of steroids in severely ill patients may be attributed to their anti-inflammatory effect, as intense inflammation is a known factor inducing lymphocytopenia and consequent increase in mortality in COVID-19.

Owing to the high possibility of the homeostatic imbalance (of the HPA axis) in patients with COVID-19, ${ }^{12-14}$ it can be argued that estimation of the serum cortisol concentrations can be helpful in deciding therapeutic strategies, more specifically for those with severe disease who have been admitted to the intensive care unit (ICU). Although application of exogenous steroids in patients with COVID-19 is being investigated intensively, the paucity of related literature suggests not much stress has been placed on the monitoring of serum cortisol. Though there is high theoretical justification, in the absence of satisfactory empirical evidence in favor, it will be advisable to address this question through scientifically designed studies designed to address whether or not serum cortisol can be used to monitor patients with COVID-19.

Along with cortisol, measuring serum adrenocorticotrophic hormone (ACTH) concentrations may provide crucial feedback on the HPA axis function in critically ill patients with COVID-19. Measuring serum ACTH concentrations becomes even more important in the case of exogenous administration of steroids, as it will have significant negative impact on ACTH secretion and consequently HPA-axis functions.,34 In a study in a mixed population of critically ill medical, surgical, trauma, and burn patients, the investigators observed that serum ACTH levels remained low/normal throughout the patients' stay in the ICU, whereas serum free cortisol levels were high both in patients with and without sepsis/septic shock. ${ }^{18}$ Unfortunately, at present, any clinical evidence on usefulness of serum ACTH monitoring in patients with COVID-19 is limited by the extreme paucity of data.

\section{Summary}

considering the possible risk of extreme serum variations and consequent impact on survival outcomes, it may be beneficial to monitor serum cortisol concentrations in critically ill patients with COVID-19. Studying patients with COVID-19 with and without administration of exogenous steroids, whether serum cortisol concentrations relate to survival outcomes, may offer important insights for the therapeutic management of the disease. $\square$
1. Jose RJ, Manuel A. COVID-19 cytokine storm: the interplay between inflammation and coagulation. Lancet Respir Med. 2020;8:e46-7.

2. Russell B, Moss C, Rigg A, Van Hemelrijck M. COVID-19 and treatment with NSAIDs and corticosteroids: should we be limiting their use in the clinical setting? Ecancermedicalscience. 2020:14:1023

3. Young A, Marsh S. Steroid use in critical care. BJA Education. 2018:18:129-34.

4. Kumar A, Kumari C, Mochan S, et al. Endocrine System. In: Vonk J, Shackelford T. (eds) Encyclopedia of Animal Cognition and Behavior. Springer, Cham 2018. doi:10.1007/978-3-319-478296_483-1.

5. Steenblock C, Todorov V, Kanczkowski W, et al. Severe acute respiratory syndrome coronavirus 2 (SARS-CoV-2) and the neuroendocrine stress axis. Mol Psychiatry. 2020:25:1611-7.

6. Boonen $\mathrm{E}$, Vervenne $\mathrm{H}$, Meersseman $\mathrm{P}$, et al. Reduced cortisol metabolism during critical illness. N Eng/ J Med. 2013:368: $1477-88$

7. RockX B, Kuiken T, Herfst S, et al. Comparative pathogenesis of COVID-19, MERS, and SARS in a nonhuman primate model. science 2020:368:1012-5.

8. Tan T, Khoo B, Mills EG, et al. Association between high serum total cortisol concentrations and mortality from COVID-19. Lancet Diabetes Endocrinol. 2020;8:659-60.

9. Sungnak W, Huang N, Bécavin C, et al. SARS-CoV-2 entry factors are highly expressed in nasal epithelial cells together with innate immune genes. Nat Med. 2020:26:681-7.

10. Muus $C$, Luecken MD, Eraslan $G$, et al Integrated analyses of single-cell atlases reveal age, gender, and smoking status associations with cell type-specific expression of mediators of SARS-COV-2 viral entry and highlights inflammatory programs in putative target cells. bioRxiv. 2020;2020.04.19.049254 [Preprint].

11. The Human Protein Atlas. SARS-CoV-2 related proteins. Available at: www.proteinatlas.org/humanproteome/sars-cov-2 (accessed August 25, 2020).

12. Téblick A, Peeters B, Langouche L, Van den Berghe G. Adrenal function and dysfunction in critically ill patients. Nat Rev Endocrinol. 2019;15:417-27.

13. Pal R. COVID-19, hypothalamo-pituitary-adrenal axis and clinical implications. Endocrine 2020; doi:10.1007/s12020-020-02325-1 [Online ahead of print].

14. Somasundaram NP, Ranathunga I, Ratnasamy $\mathrm{V}$, et al. The impact of SARS-Cov-2 virus infection on the endocrine system. J Endocr SOC. 2020;4:bvaa082.

15. Lin LL, GU HY, Luo J, et al. Impact and beneficial critical points of clinical outcome in corticosteroid management of adult patients with sepsis: meta-analysis and grade assessment Front Pharmacol. 2019:10:1101.

16. RECOVERY Collaborative Group, Horby P, Lim WS, et al. Dexamethasone in hospitalized patients with covid-19preliminary report. N Eng/ J Med. 2020; doi:10.1056/NEJMoa2021436. [Online ahead of print].

17. Minneci PC, Deans KJ, Eichacker PQ, Natanson C. The effects of steroids during sepsis depend on dose and severity of illness: an updated meta-analysis. Clin Microbiol Infect. 2009;15:308-18.

18. Peeters $B$, Meersseman $P$ Vander Perre $S$, et al. Adrenocortical

function during prolonged critical illness and beyond: a prospective observational study. Intensive Care Med. 2018;44:1720-9. 